\title{
Low-Grade Endometrial Stromal Sarcoma and Uterine Adenosarcoma: A Comparison of Clinical Manifestations and Outcomes
}

\author{
Yanyan Zhang, Yan Li, Huifang Huang, Jiaxin Yang, Ming Wu, Ying Jin ${ }^{\bowtie *}$, Lingya Pan ${ }^{\bowtie *}$ \\ Department of Obstetrics and Gynecology, Peking Union Medical College Hospital, Chinese Academy of Medical Sciences \& Peking Union Medical College, \\ Beijing, China. \\ * These authors contributed equally to this work \\ $\square$ Corresponding authors: Lingya Pan, Department of Obstetrics and Gynecology, Peking Union Medical College Hospital, Chinese Academy of Medical \\ Sciences \& Peking Union Medical College, 1 Shuai Fu Yuan, Wang Fu Jing Street, Beijing 100730, China Tel: +86-10-65296203, Fax: +86-10-65124875, Email: \\ panly@pumch.cn and Ying Jin, Department of Obstetrics and Gynecology, Peking Union Medical College Hospital, Chinese Academy of Medical Sciences \& \\ Peking Union Medical College, 1 Shuai Fu Yuan, Wang Fu Jing Street, Beijing 100730, China Tel: +86-10-69155731, Fax: +86-10-65124875, Email: \\ jinying@pumch.cn
}

(c) Ivyspring International Publisher. This is an open access article distributed under the terms of the Creative Commons Attribution (CC BY-NC) license (https:// creativecommons.org/licenses/by-nc/4.0/). See http://ivyspring.com/terms for full terms and conditions.

Received: 2018.10.15; Accepted: 2019.05.12; Published: 2019.06.05

\begin{abstract}
Objective: Our study aimed to assess factors associated with progression free survival (PFS) and overall survival (OS) in low grade endometrial stromal sarcoma (LG-ESS) and uterine adenosarcoma, and to determine the differences in clinical manifestations and outcomes between the two diseases.

Methods: A total of 132 patients were enrolled in this retrospective study at Peking Union Medical College Hospital from 1998 to 2016. The associations of clinical and pathological factors with PFS and OS were evaluated.

Results: Of the 132 included patients, 104 had LG-ESS and 28 had uterine adenosarcoma. All patients were followed up for at least 1.5 years. There were significant differences between LG-ESS and uterine adenosarcoma in terms of age distribution $(41.05 \pm 10.5$ vs $46.11 \pm 14.96$ years, $P=0.042)$, delivery time (nulliparity $=0$ : $18.27 \%$ vs $35.71 \%, P=0.046)$, history of the uterine leiomyoma $(65.38 \%$ vs $39.29 \%$, $\mathrm{P}=0.012)$, and polypoid tumor growth $(14.42 \%$ vs $60.71 \%, \mathrm{P}=0.007)$. According to the pathological findings, the proportion of uterine adenosarcoma patients with uterine leiomyoma (60.71\%) was significantly higher than that for the LG-ESS patients $(32.69 \%)(P=0.007)$. Uterine adenosarcoma seemed to be associated with longer PFS and OS than LG-ESS (PFS: $42.69 \pm 29.94$ vs $50.50 \pm 40.50$ months; OS: $58.72 \pm 37.29$ vs $69.46 \pm 47.58$ months), but the differences were not statistically significant. Multivariate Cox regression showed that age, menopause, history of uterine leiomyoma, stage, and hormone therapy were independent risk factors with respect to PFS, whereas age and stage were risk factors affecting OS in LG-ESS patient. Peritoneal lavage cytology and radiotherapy were risk factors affecting PFS and peritoneal lavage cytology for OS in patients with uterine adenosarcoma.

Conclusion: The patients with advanced LG-ESS had poor prognosis. Age and history of uterine leiomyoma were associated with poor PFS, while menopause and hormone therapy were protective factors associated with improved PFS in patients with LG-ESS. Peritoneal lavage cytology and radiotherapy did not improve prognosis of uterine adenosarcoma.
\end{abstract}

Key words: low-grade endometrial stromal sarcoma, uterine adenosarcoma, clinical manifestations, prognosis

\section{Introduction}

Uterine adenosarcoma, which accounts for $5-10 \%$ of uterine sarcoma, comprises a group of mixed mesenchymal tumors most commonly arising from the endometrium, benign glandular epithelium and low-grade sarcoma [1-2]. In these cancers, a benign epithelial component exists together with a malignant 
stromal component that resembles low-grade endometrial stromal (LG-ESS) [3]. Microscopically, LG-ESS is composed of well-differentiated endometrial stromal cells [4-5], and it is histologically similar to adenosarcoma.

Both LG-ESS and uterine adenosarcoma have malignant stromal components, and their clinical outcomes are also similar. They are generally characterized by an indolent course and a favorable prognosis. Clinical manifestations, of LG-ESS and uterine adenosarcoma usually involve the uterine cavity or intermuscular nodule, or a polypoid neoplasm in the cervix. The standard treatment is total hysterectomy and bilateral ovariectomy.

Although LG-ESS and uterine adenosarcoma have similar clinical manifestations, pathological features and principles and methods of surgery, they have different biological characteristics and represent two different pathological types; thus, the choice of adjuvant therapy and its influence on prognosis are not the same. Uterine adenosarcoma is commonly viewed as one of the more chemo-sensitive soft tissue sarcoma subtypes, and so the choice of chemotherapy follows a soft tissue sarcoma paradigm [3], with single-agent doxorubicin as the standard first-line therapy $[3,6]$. The hormonal estrogen receptors (ER) and progesterone receptor (PR) are frequently expressed in patients with LG-ESS and may be exploited for therapeutic benefit [7]. Therefore, LG-ESS is most often treated with anti-estrogen therapy [8-9]. Some success has been reported with gonadotropin-releasing hormone (GnRH) analogs and aromatase inhibitors [8, 10-11].

The purpose of this study is to deepen and update our understanding of these diseases and determine the factors influencing the prognosis. To derive robust inferences, data of different diseases were analyzed separately [12].

\section{Patients and Methods}

This was a single-center study conducted in the Gynecology and Obstetrics department of Peking Union Medical College Hospital (PUMCH). The study received approval from the institutional review board of $\mathrm{PUMCH}$ and all patients provided informed consent on admission to PUMCH.

Patients were eligible for this study if they had a definite pathological diagnosis of LG-ESS $(n=104)$ or uterine adenosarcoma $(n=28)$ and were treated at our hospital between 1998 and 2016. Histological classification was based on the 2014 World Health Organization (WHO) classification. Demographic, clinicopathological, treatment, and outcomes data were obtained from medical records and from the telephone follow-up survey. We excluded patients with other neoplastic diseases and those for whom detailed follow-up data were not available $(n=4)$. All patients were Asian women.

Each patient's basic information included age, menopause status, parity, history of uterine leiomyoma (based on previous imaging data), initial presentation, hormone therapy, chemotherapy, radiotherapy, and International Federation of Gynecology and Obstetrics (FIGO) stage. Stage of disease was determined according to the 2009 FIGO staging for uterine sarcomas. All patients were followed up for at least 18 months.

Surgery is the standard treatment. Complete surgery for ESS and uterine adenosarcoma is defined as total hysterectomy with bilateral salpingooophorectomy in stage I and removal of enlarged nodes and debulking of obvious extra uterine disease in the advanced stages II to IV [4]. In our study, surgical procedures included complete surgery, hysterectomy, myomectomy or lesionectomy.

Adjuvant therapies include radiotherapy, chemoradiotherapy and endocrine therapy. Radiation dose and location were based on the patient's condition, so there is no universal standard. The chemotherapy plans PEB (cisplatin/epirubicin/ bleomycin), PE (cisplatin/epirubicin), PEI (cisplatin/ epirubicin/ifosfamide), TI (taxol/ifosfamide) have been used. Endocrine therapy includes megestrol acetate, tamoxifen, and GnRH-a.

We assessed the relationships among clinical expressions, pathological features, treatment and disease outcome. Outcome indicators included PFS and OS. PFS is defined as the time from treatment to relapse or to final follow-up, while OS is defined as the time from the beginning of treatment to death or to the last follow-up. Age, menopause status, history of uterine leiomyoma, FIGO stage (stage I vs stage II-IV), lymphadenectomy (no vs negative vs positive), Peritoneal lavage cytology (no vs negative for free cancer cells vs positive for free cancer cells), lymphovascular space invasion (LVSI), endometriosis, uterine fibroids, chemotherapy, radiotherapy, and hormone therapy were independent variables for evaluating relapse and survival.

The characteristics of patients are summarized in Table 1 and Table 2.

\section{Statistical analysis}

Statistical analysis was performed with SAS 9.4 software (Cary, NC). Continuous variables are represented by mean \pm standard or median (minimum-maximum) and analysis of variance (ANOVA) was performed. Categorical data were expressed by sample number (proportion \%) and the 
X2 test was used. Cox regression analysis was carried out by the stepwise method. After adjusting for the influence of other factors, a multivariate Cox regression model was constructed based on whether endpoint event occurred and on the time of the occurrence of the endpoint event. Statistical significance was assumed at $\mathrm{P}<0.05$.

Table 1. Patient Characteristics.

\begin{tabular}{|c|c|c|}
\hline Variable & $\begin{array}{l}\text { LG-ESS } \\
\mathrm{N}=104, \text { No. } \\
(\%)\end{array}$ & $\begin{array}{l}\text { Uterine } \\
\text { adenosarcoma } \\
\mathrm{N}=28, \text { No. }(\%)\end{array}$ \\
\hline Age (years)* & $41.05 \pm 10.5$ & $46.11 \pm 14.96$ \\
\hline \multicolumn{3}{|l|}{ Menopause * } \\
\hline Yes & $17(16.35)$ & $8(28.57)$ \\
\hline No & $87(83.65)$ & $20(71.43)$ \\
\hline \multicolumn{3}{|l|}{ Parity } \\
\hline $0^{*}$ & $19(18.27)$ & $10(35.71)$ \\
\hline 1 & $65(62.50)$ & $10(35.71)$ \\
\hline$>1$ & $16(15.38)$ & $8(28.57)$ \\
\hline unknown & $4(3.85)$ & $0(0)$ \\
\hline \multicolumn{3}{|l|}{ History of uterine leiomyoma* } \\
\hline Yes & $68(65.38)$ & 11(39.29) \\
\hline No & $36(34.62)$ & $17(60.71)$ \\
\hline \multicolumn{3}{|l|}{ Initial presentation } \\
\hline Menorrhagia & $49(47.12)$ & $11(39.29)$ \\
\hline Postmenopausal bleeding & $3(2.88)$ & $8(28.57)$ \\
\hline Abdominal discomfort & $22(21.15)$ & $2(7.14 \%)$ \\
\hline Frequent micturition or dysporia & $7(6.73)$ & $4(14.29)$ \\
\hline Algopareunia or contact bleeding & $3(2.88)$ & $2(7.14)$ \\
\hline \multicolumn{3}{|l|}{ Tumor grew like a polyp* } \\
\hline Yes & $15(14.42)$ & $17(60.71)$ \\
\hline No & $89(85.58)$ & $11(39.29)$ \\
\hline \multicolumn{3}{|l|}{ Surgical procedure } \\
\hline Complete surgery & $91(87.50)$ & $24(85.71)$ \\
\hline Hysterectomy & $5(4.81)$ & $0(0)$ \\
\hline Myomectomy/lesionectomy & $8(7.69)$ & $4(14.29)$ \\
\hline \multicolumn{3}{|l|}{ FIGO Stage (2009) } \\
\hline$I$ & $76(73.08)$ & $23(82.14)$ \\
\hline II & $10(9.62)$ & $3(10.71)$ \\
\hline III & $9(8.65)$ & $2(7.14)$ \\
\hline IV & $9(8.65)$ & $0(0)$ \\
\hline Adjuvant therapy & $79(75.96)$ & $16(57.14)$ \\
\hline Chemotherapy & $5(4.81)$ & $5(17.86)$ \\
\hline Radiotherapy & $6(5.77)$ & $3(10.71)$ \\
\hline Endocrinotherapy & $33(31.73)$ & $3(10.71)$ \\
\hline Chemotherapy+radiotherapy & $6(5.77)$ & $2(7.14)$ \\
\hline Chemotherapy+endocrinotherapy & $10(9.62)$ & $1(3.57)$ \\
\hline Radiotherapy+endocrinotherapy & $16(15.39)$ & $1(3.57)$ \\
\hline Chemotherapy + radiotherapy+endocrinotherapy & $3(2.89)$ & $2(7.14)$ \\
\hline PFS (month) & $42.69 \pm 29.94$ & $50.50 \pm 40.50$ \\
\hline$\geq 5$ years & $32(58.18)$ & $10(58.82)$ \\
\hline OS (month) & $58.72 \pm 37.29$ & $69.46 \pm 47.58$ \\
\hline$\geq 5$ years & $48(87.27)$ & $13(76.47)$ \\
\hline Recurrence & $36(40.38)$ & $10(35.71)$ \\
\hline Deaths & $10(9.60)$ & $3(10.71)$ \\
\hline
\end{tabular}

\section{Treatment}

Of the patients with LG-ESS, 91(87.50\%) underwent complete surgery. Five patients $(4.81 \%)$ received hysterectomy, of whom four were in stage I and one was in stage II. Two of these patients received radiotherapy and two received endocrinotherapy; the follow-up time ranged from 34 to 96 months and no recurrence occurred during the follow-up period. Eight patients $(7.69 \%)$ received myomectomy or lesionectomy; seven of them were in stage I and received endocrinotherapy; the follow-up time ranged from 10 to 60 months and no recurrence occurred during the follow-up period. The other patient was in stage IV, with an age at diagnosis of 72 years, and died 12 months after diagnosis). 
Seventy-nine patients $(75.96 \%)$ received adjuvant therapy. (Table 1)

Of the patients with uterine adenosarcoma, 24 $(85.71 \%)$ underwent complete surgery and four patients $(14.29 \%)$ received myomectomy or lesionectomy. Sixteen patients $(57.14 \%)$ received adjuvant therapy. The four patients who did not receive complete surgery were all in stage $\mathrm{I}$, and were 19, 31, 32 and 33-years-old. One of them was treated with chemotherapy. The follow-up time ranged from 24 to 48 months and no recurrence occurred in any of these four patients during the follow-up period. (Table 1)

\section{Pathological findings}

In patients with LG-ESS, lymph node dissection was performed in $32(30.77 \%)$ patients, two $(1.92 \%)$ of whom had lymph node metastasis. Six patients $(21.43 \%)$ with uterine adenosarcoma underwent lymph node dissection; none of them had lymph node metastasis. The positive rate of free cancer cells in pre-operative abdominal washings in LG-ESS and uterine adenosarcoma was $3.03 \%(1 / 33)$ and $33.33 \%(2 / 6)$, respectively. Endometriosis occurred in $29.81 \%$ of patients with LG-ESS and $35.71 \%$ of patients

A

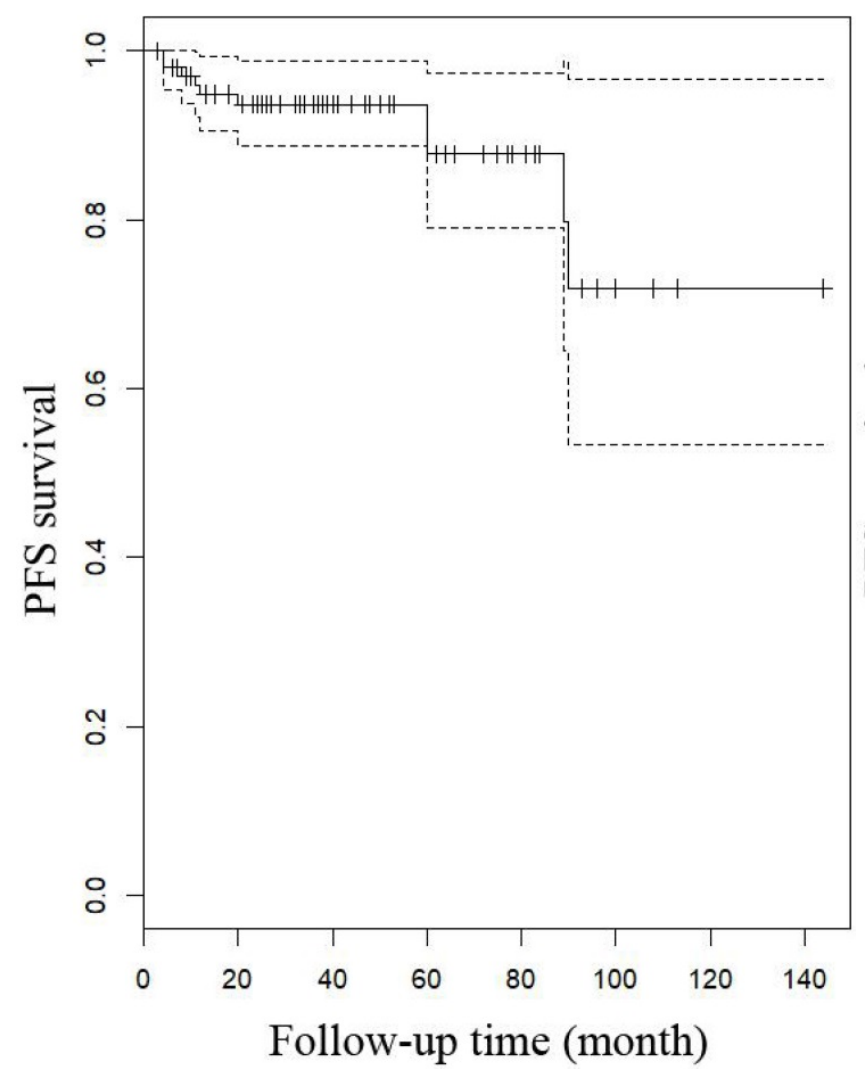

with uterine adenosarcoma. Compared with patients with LG-ESS $(32.69 \%)$, more than half of uterine adenosarcoma patients $(60.71 \%)$ had uterine leiomyoma $(\mathrm{P}=0.021)$. Twenty-two patients $(21.15 \%)$ with LG-ESS and three patients $(10.71 \%)$ with uterine adenosarcoma had LVSI. (Table 2)

\section{Outcomes and prognostic factors}

The median follow-up time for LG-ESS and uterine adenosarcoma were 51 months (range: 8-216 months) and 53.5 months (range: 7-158 months), respectively. In patients with LG-ESS, the median PFS was 37.5 months (range: 3-144 months) and 36 (34.62\%) patients relapsed. The median OS was 50 months (range: 8-216 months) and 10 (9.60\%) patients died from the disease. Five-year survival rates were $58.18 \%$ for PFS and $58.82 \%$ for OS. In patients with uterine adenosarcoma, the median PFS was 42 months (range: 2-118 months) and eight (26.00\%) patients relapsed. The median OS was 53.5 months (range: $7-158$ months) and three $(10.71 \%)$ patients died from the disease. The five years rates were $87.27 \%$ for PFS and $76.47 \%$ for OS. There were no differences in outcomes between the two diseases (Figure 1 and Figure 4).

B

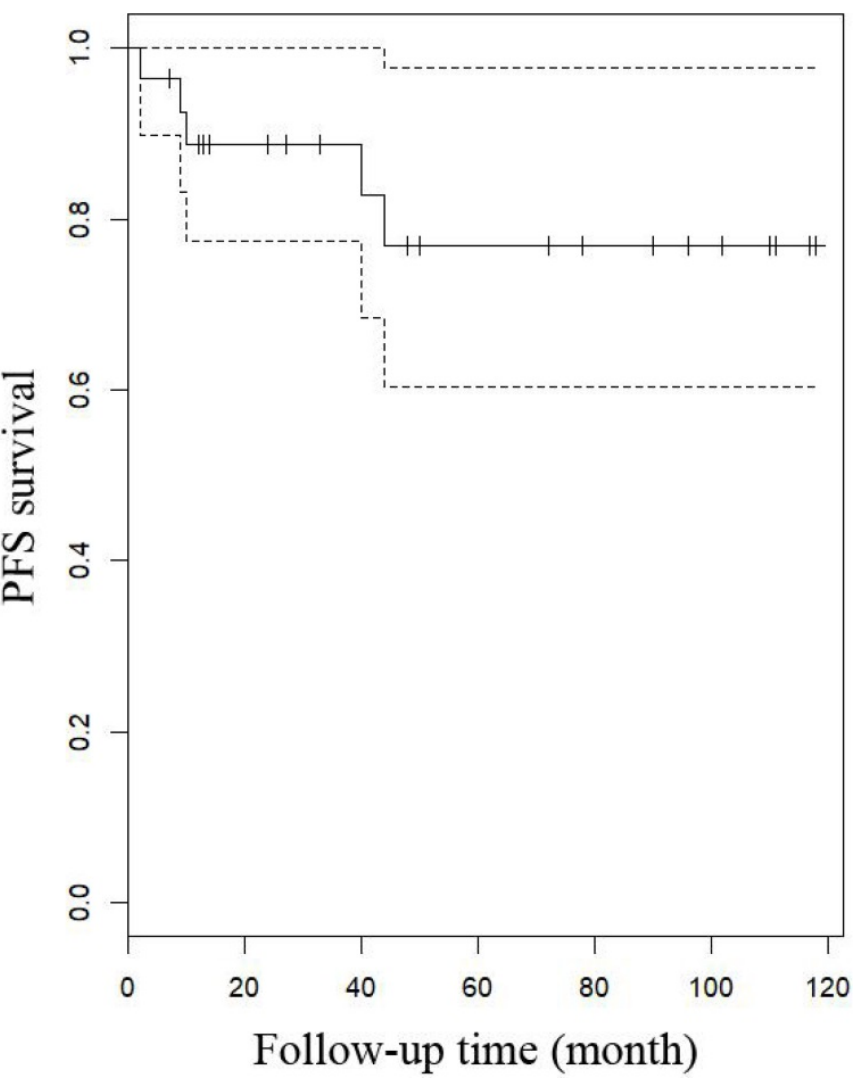

Figure 1. PFS of patients (A) with LG-ESS and (B) with uterine adenosarcoma. 
A

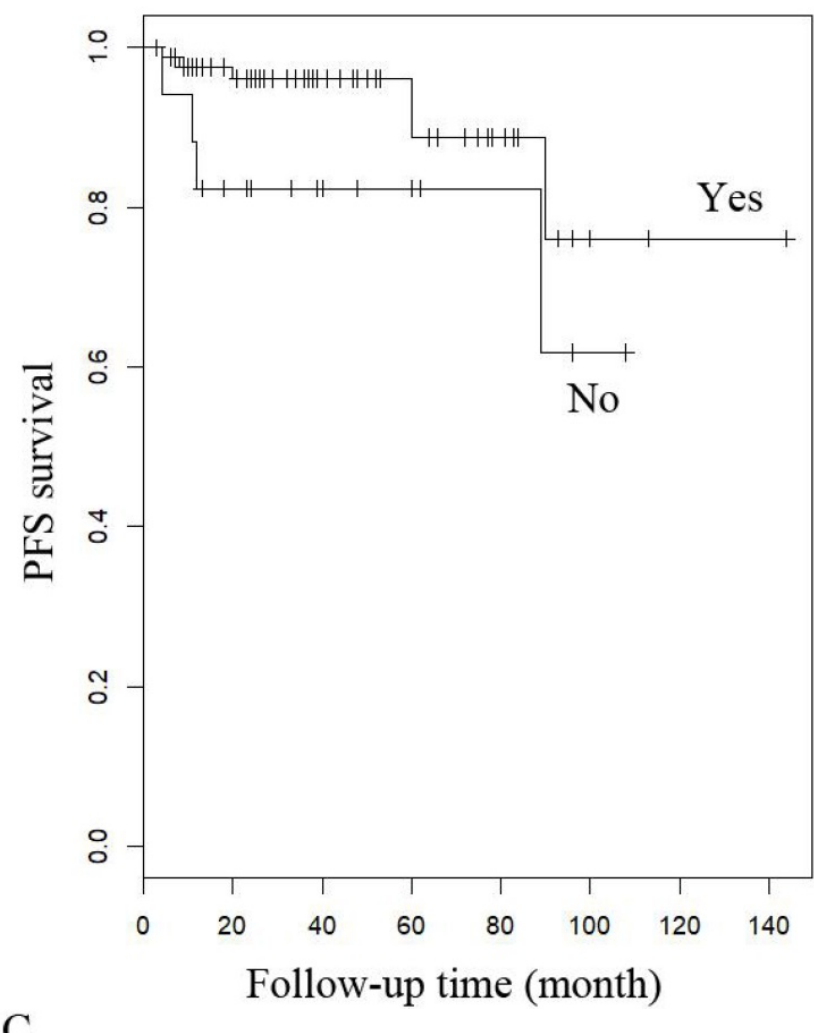

C

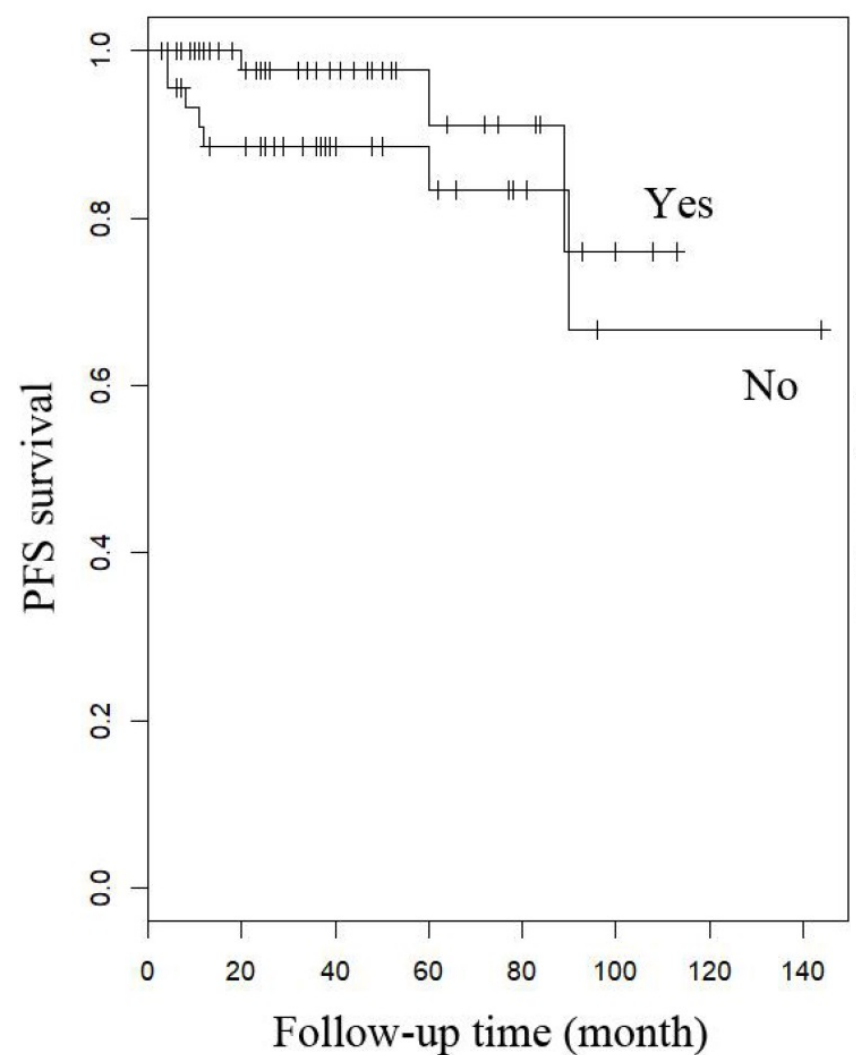

B

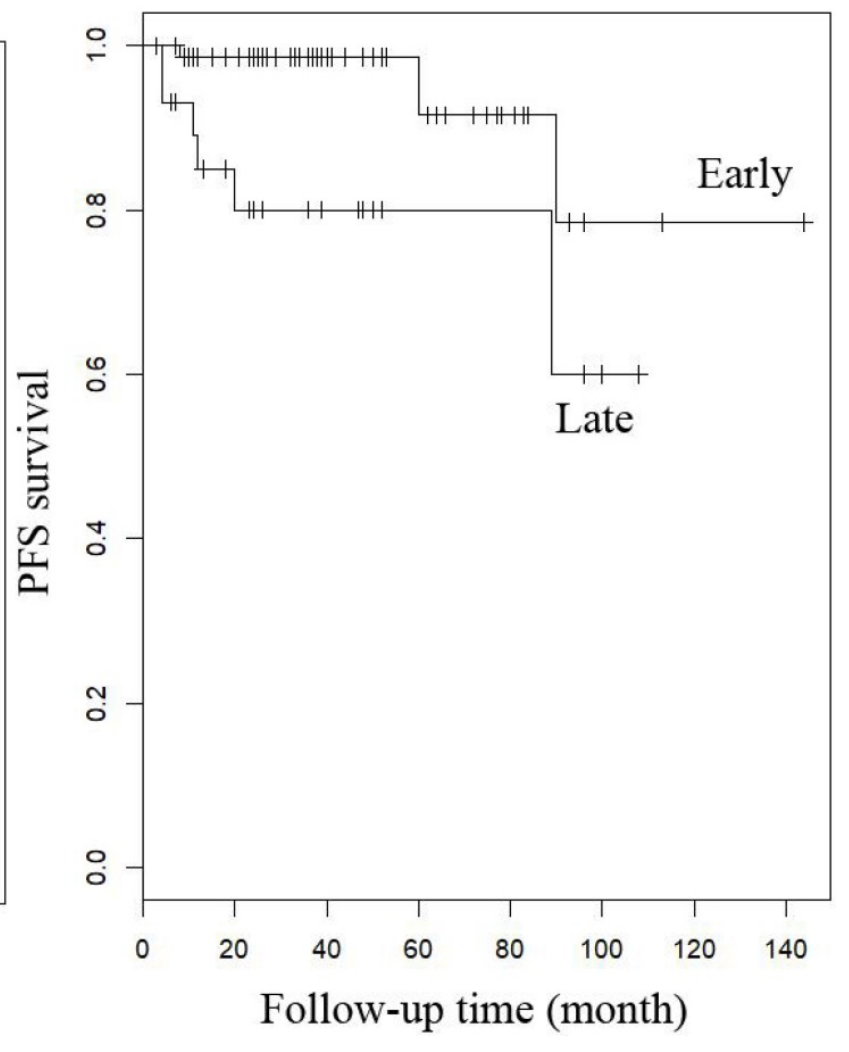

D

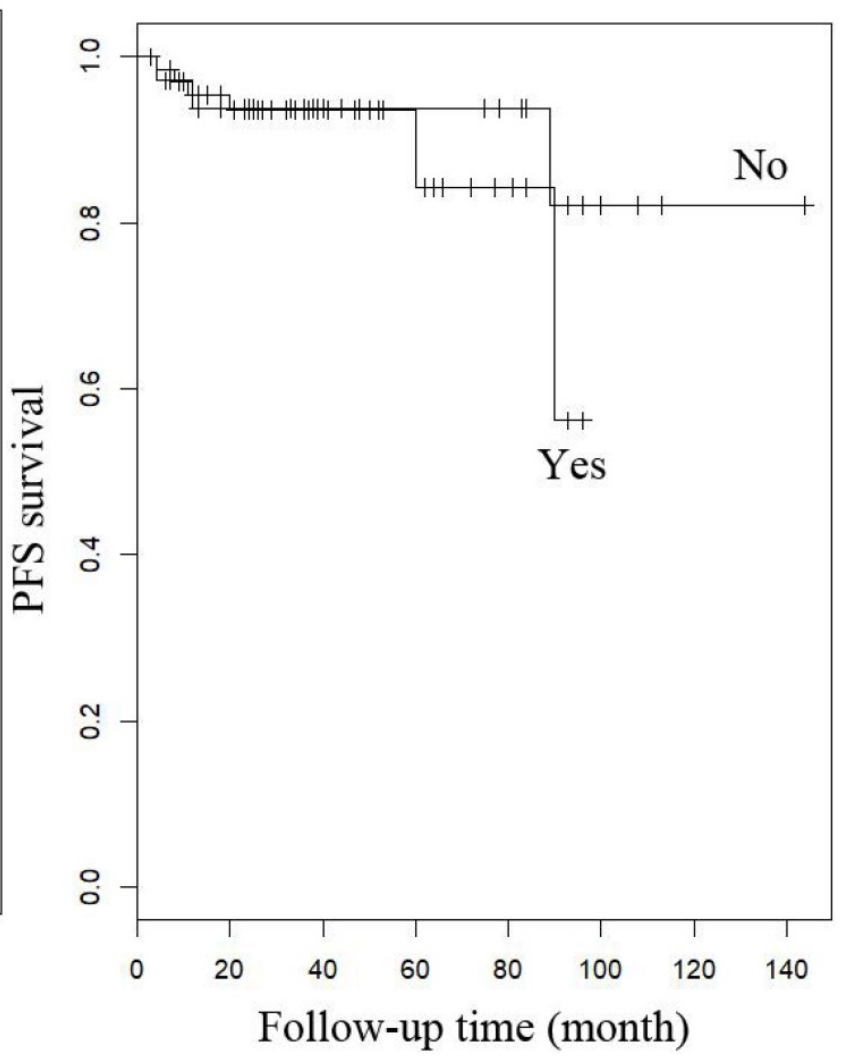

Figure 2. PFS of patients with LG-ESS by (A) menopause (yes or no), (B) stage: (early or late), (C) hormone therapy (with or without), and (D) history of uterine leiomyoma. 
A

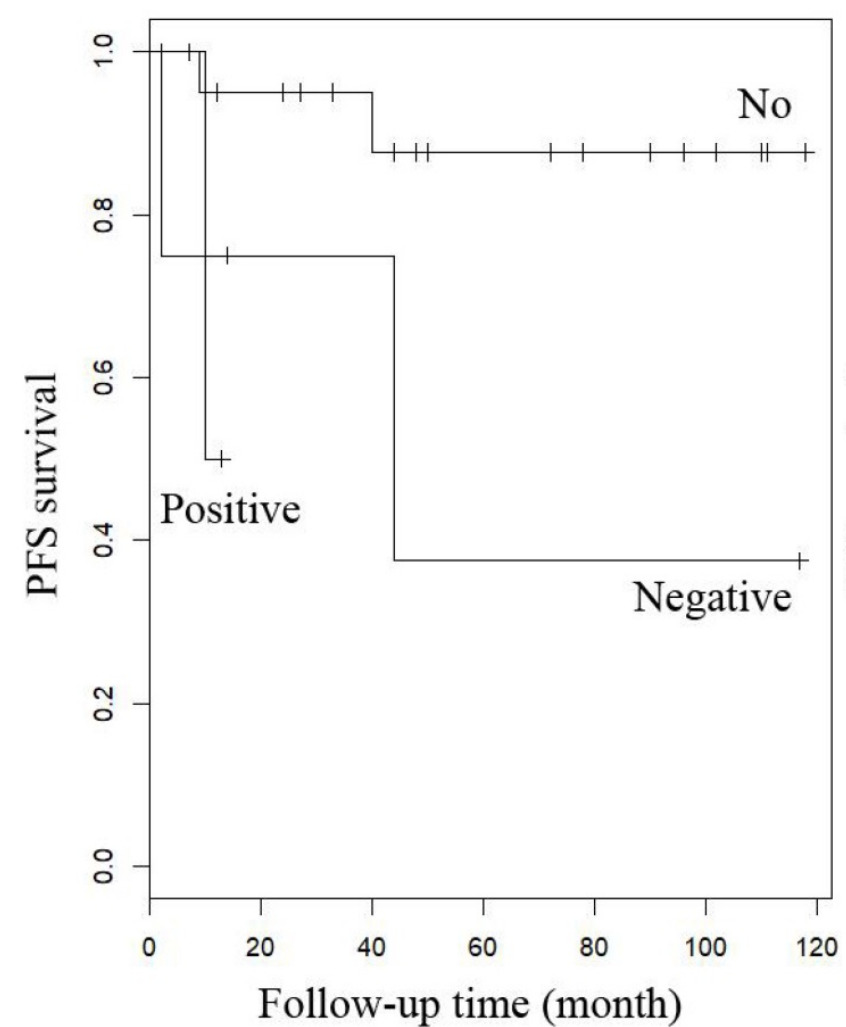

B

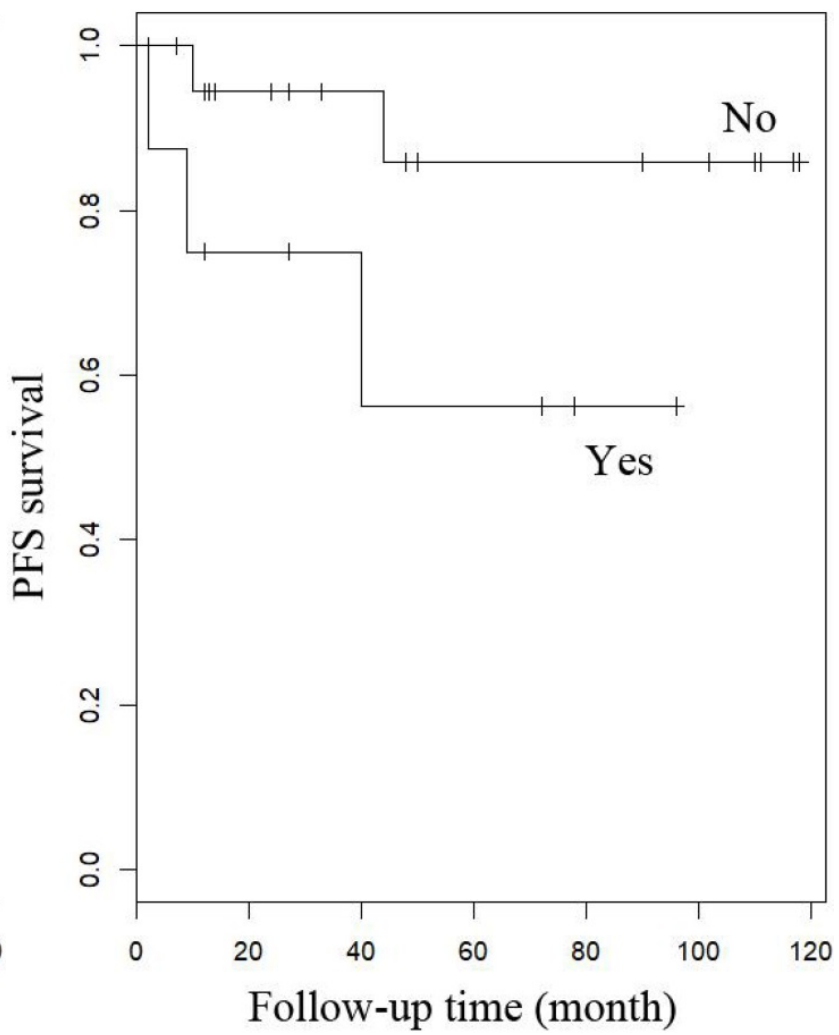

Figure 3. PFS of patients with uterine adenosarcoma (A) with (negative or positive) or without peritoneal lavage cytology, and (B) with or without Radiotherapy.

A

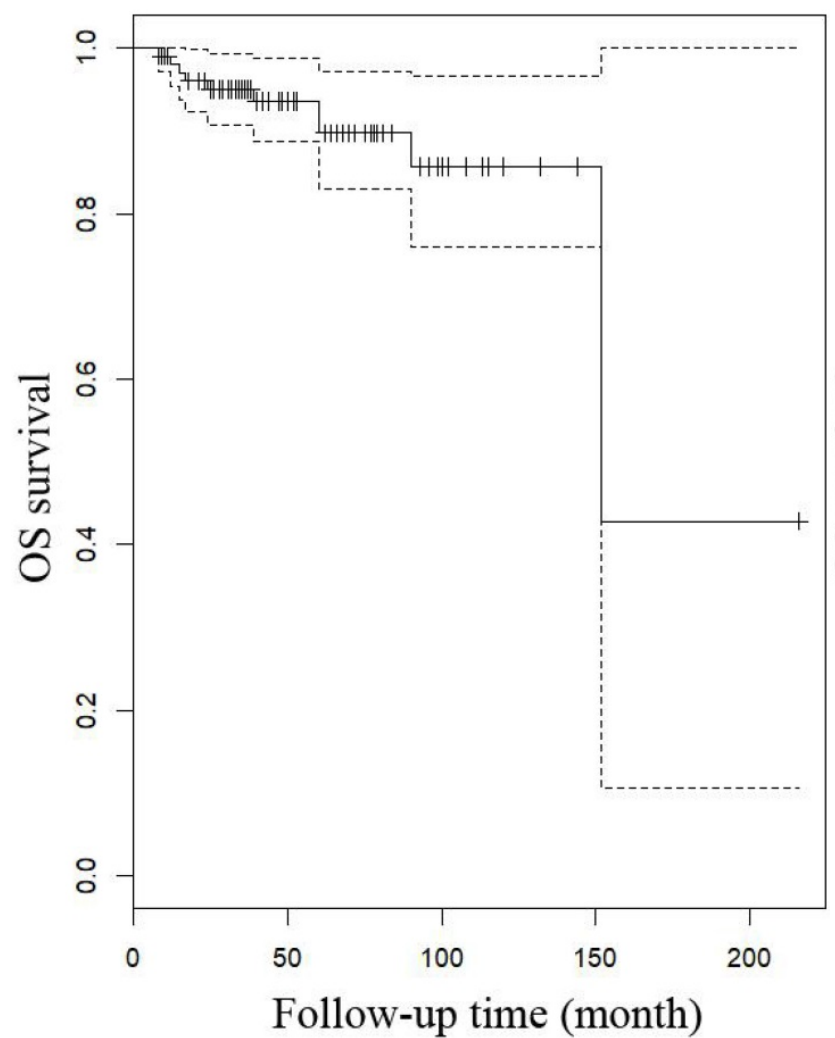

B

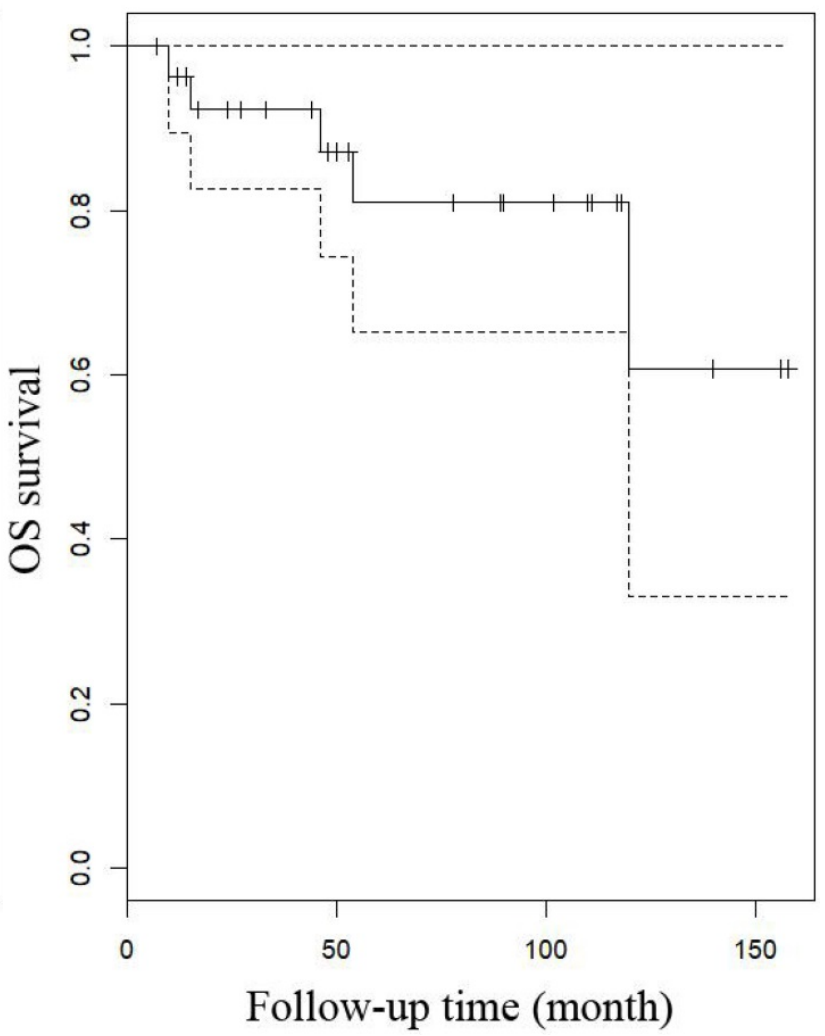

Figure 4. OS of patients (A) patients with LG-ESS and (B) with uterine adenosarcoma 
A

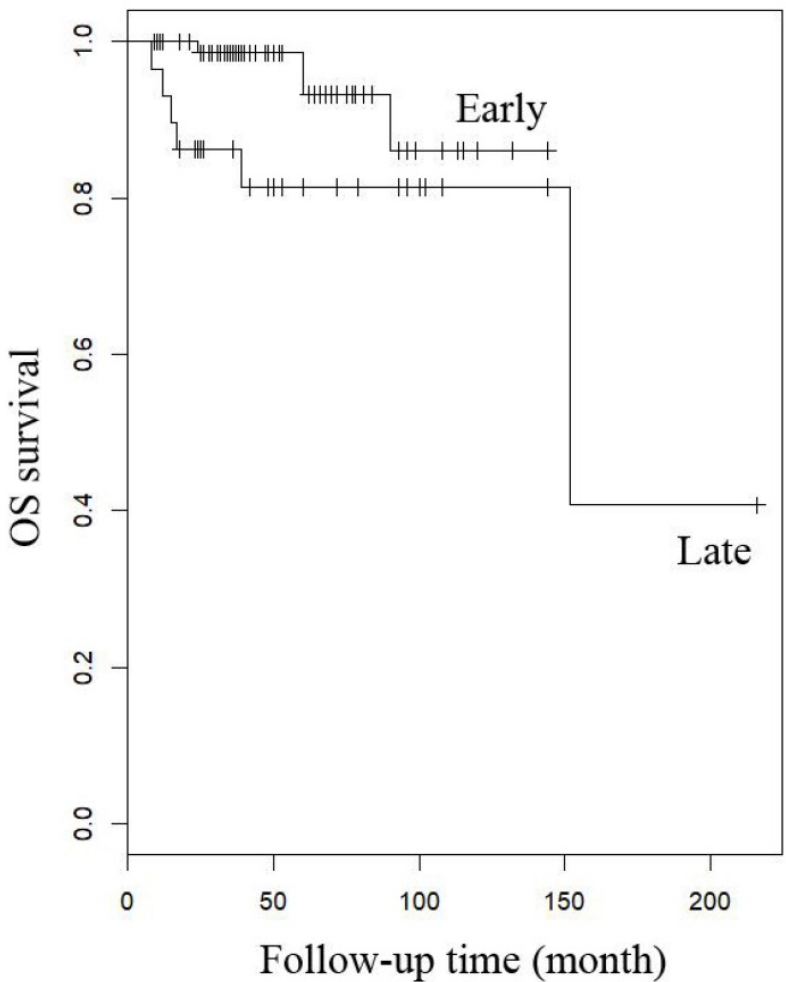

B

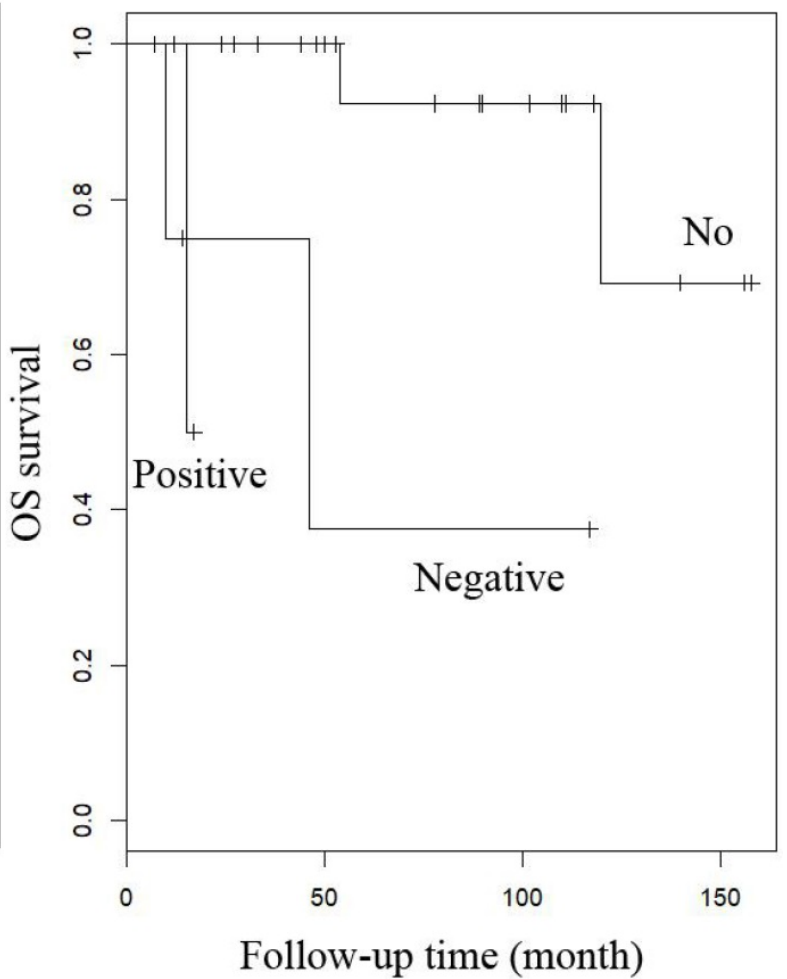

Figure 5. OS of patients (A) LG-ESS in early or late stage and (B) uterine adenosarcoma with (negative or positive) or without peritoneal lavage cytology.

When other factors were corrected, multivariate Cox regression showed that older age at diagnosis (hazard ratio (HR) 1.27, 95\% CI 1.07-1.5, $\mathrm{P}=0.006$; the probability of relapse increased by 1.27 times for every 1 year increase in age), history of uterine leiomyoma (HR 36.89, 95\% CI 2.1-646.93, $\mathrm{p}=0.014$ ), and late stage (HR 163.86, 95\% CI 7-3837.4, p=0.002) were independent risk factors, while menopause (HR $0.001,95 \%$ CI $0.001-0.66, \mathrm{p}=0.031)$ and hormone therapy (HR 0.14, 95\% CI 0.02-0.9, p=0.039) were protective factors with respect to PFS in LG-ESS patients (Figure 2). Peritoneal lavage cytology (HR $22.71,95 \%$ CI 1.64-315.33, p=0.020) and radiotherapy (HR 81.17, 95\% CI 1.16-5687.58, p=0.043) were independent risk factors affecting PFS in patients with uterine adenosarcoma (Figure 3). Older age at diagnosis (HR 1.13, 95\% CI 1.03-1.23, p=0.007) and late stage (HR 7.17, 95\% CI 1.53-33.56, $\mathrm{p}=0.012$ ) were independent risk factors for OS of LG-ESS patients and peritoneal lavage cytology (HR 7.32, 95\% CI 1.49-36.01, $\mathrm{p}=0.014)$ was an independent risk factor affecting OS of uterine adenosarcoma patients (Figure $5)$.

\section{Discussion}

In this study, we confirmed that patients with LG-ESS and uterine adenosarcoma had similar outcomes. However, they had different clinical manifestations and prognostic factors. LG-ESS was often misdiagnosed as uterine fibroids, the most common symptom being irregular menstruation, only $17.65 \%$ of the postmenopausal patients in our study presented with postmenopausal bleeding. Multivariate Cox regression showed that older age at diagnosis, history of uterine leiomyoma, and late stage were independent risk factors, while menopause and hormone therapy were protective factors with respect to PFS of LG-ESS patients. Older age at diagnosis and late stage were independent risk factors for OS. Compared with patients with LG-ESS, uterine adenosarcoma patients were older at diagnosis and their tumors often grew like polyps. Interestingly, more than half of the uterine adenosarcoma patients and $32.69 \%$ of the LG-ESS patients had uterine fibroids. All the postmenopausal patients with uterine adenosarcoma presented with vaginal bleeding. Cox regression showed that peritoneal lavage cytology and radiotherapy were independent risk factors affecting PFS, while peritoneal lavage cytology was an independent risk factor affecting OS in patients with uterine adenosarcoma.

ESS is a rare pathological type of uterine sarcoma, which is often misdiagnosed as benign uterine diseases because of a lack of characteristic imaging and clinical manifestations. Most of ESS 
patients were misdiagnosed as having uterine leiomyoma before surgery, which delayed treatment; this may contribute to the poor prognosis of LG-ESS patients with a history of uterine fibroids. In patients with uterine adenosarcoma, tumors often grow like polyps. Therefore, the disease is often found early owing to neoplasms of the vagina and abnormal vaginal bleeding.

Standard treatment consists of hysterectomy with bilateral salpingo-oophorectomy in the early stage (stage I) and tumor cell reduction surgery in advanced stages (stage II-IV) [4]. Whether to keep ovaries in patients with LG-ESS and uterine adenosarcoma is still under debate. In our cohort, of 17 patients who underwent ovarian preservation surgery, one was 72 years old and diagnosed as stage $\mathrm{IV}$, this patient died 12 months after diagnosis. Of the remaining 16 patients who presented at an early age (range: 15-36 years), two patients were in stage II and 14 were in stage I. Ten of them received hormonotherapy and two received chemotherapy; none of the 16 patients relapsed during the follow-up period. In agreement with Carroll et al. [13], we believe that local resection or with or without adjuvant therapy can be considered for young patients in the early stages who desire future fertility.

In this study, 12 patients received myomectomy or lesionectomy, 11 of who had no recurrence during the follow-up period. We found no differences in outcomes between laparoscopic myomectomy and abdominal myomectomy. The US Food and Drug Administration released a warning in 2014 that the use of a laparoscopic electric morcellator in patients with unpredictable uterine sarcoma can increase the risk of dissemination of tumor tissue in the abdominopelvic cavity, particularly affecting long-term survival rates [14-15,27]. In Gao' study [15] showed that fibroid morcellation during laparoscopic surgery (including laparoscopic, transvaginal and transabdominal approaches) had no significant impact on PFS and OS, while grade was a significant risk factor for the prognosis of patients with uterine sarcoma, consistent with our results.

The prognostic significance of lymph nodal metastasis and complete lymphadenectomy is still a matter of debate [4, 10, 16-17]. According to multivariate Cox regression analysis, we found that lymph node dissection did not improve the prognosis of patients with LG-ESS or uterine adenosarcoma. Rates of lymph nodes positivity in patients with LG-ESS and uterine adenosarcoma were only $6.25 \%$ and $0 \%$ respectively. However, Seagle's study [18] found that lymph node positivity showed a weak trend toward a strongly negative prognostic association, and those women with high-grade ESS and no surgical node evaluation had significantly decreased survival. A meta-analysis by Si's meta-analysis [19] suggested that lymphadenectomy has little prognostic or therapeutic benefit in patients with uterine sarcoma. Systematic lymphadenectomy may not be recommended in patients with uterine adenosarcoma or ESS unless the patient has obvious extrauterine involvement, clinically suspicious enlarged nodes, or advanced sarcoma [12].

In addition to surgery, hormone therapy, radiotherapy and chemotherapy are important adjuvant treatments for LG-ESS and uterine adenosarcoma, but their effects need to be identified and discussed. Clinical trials have shown no definite survival benefit of adjuvant radiotherapy, although they have been hampered by the rarity and heterogeneity of these disease types [20]. Adjuvant hormonal suppression with high dose progestins, aromatase inhibitors or GnRH-agonists may have a survival benefit for patients with LG-ESS. The efficacy of hormonal therapy has been well documented in recurrent and advanced disease but has yet to be adopted as routine practice in the adjuvant setting, despite multiple series suggesting a benefit [21-26]. Almost $80 \%$ of LG-ESS patients express ER alpha and PgR, providing an opportunity for adjuvant endocrine therapy [13]. Our results indicated that hormone therapy could only improve PFS of LG-ESS, and that menopause and age were protective factors with respect to PFS and OS, respectively. Pathological analyses of uterine leiomyosarcoma have identified ER positivity in approximately $80 \%$ of cases and progesterone receptor positivity in $65-80 \%$ of cases [13], but we did not find any benefit of hormone therapy for patients with uterine adenosarcoma. Nor did we find that chemotherapy and radiotherapy could improve the outcome of the disease. Our conclusions are consistent with previous studies, in which no survival benefit was seen in uterine adenosarcoma patients who received adjuvant pelvic radiotherapy [2]. Based on our analysis, peritoneal lavage cytology was associated with poor prognosis in patients with uterine adenosarcoma. This might be due to the spread of the tumor cells by incomplete irrigation of the abdominal cavity, or to the skewing produced by our small number of cases, so we need to expand the sample for further study. The optimal adjuvant treatment is still unclear.

Our study had some limitations: As it was a single center retrospective study with limited sample size, the conclusions may be affected by the number of cases. Some patients received treatment in other hospitals before they came to ours, which may limit the generalizability of the results and predispose them to selection bias. Further multicenter trials with larger 
numbers of patients are required in to confirm our results.

In conclusion, we should be on the alert for patients with suspected uterine myoma or endometrial polyps. In the absence of a clear diagnosis, we should take curettage or biopsy to confirm the diagnosis. Unless the patient has obvious extrauterine involvement, clinically suspicious enlarged nodes, or advanced sarcomas, lymphadenectomy is necessary. For LG-ESS, complete surgery is the main treatment, and adjuvant hormone therapy can improve the patient's prognosis. Owing to the lack of effective adjuvant therapy for uterine adenosarcoma, complete surgery should be carried out.

\section{Abbreviations}

LG-ESS: low-grade endometrial stromal sarcoma; PFS: progression-free survival; OS: overall survival; ER: estrogen receptor; PR: progesterone receptor; GnRH: gonadotropin-releasing hormone; PUMCH: Peking Union Medical College Hospital; WHO: World Health Organization; FIGO: International Federation of Gynecology and Obstetrics; LVSI: lymphovascular space invasion; HR: hazard ratio.

\section{Competing Interests}

The authors have declared that no competing interest exists.

\section{References}

1. Clement PB, Scully RE. Mullerian adenosarcoma of the uterus: a clinicopathologic analysis of 100 cases with a review of the literature. Human Pathology. 1990; 21(4): 363-81.

2. Nathenson MJ, Conley AP, Lin $\mathrm{H}$, et al. Treatment of recurrent or metastatic uterine adenosarcoma. Sarcoma. 2017; 2017: 4680273.

3. Benson $C$, Miah AB. Uterine sarcoma - current perspectives. Int J Womens Health. 2017; 9: 597-606.

4. Agarwal R, Rajanbabu A, Nair IR, et al. Endometrial stromal sarcoma-A retrospective analysis of factors affecting recurrence. Eur J Obstet Gynecol Reprod Biol. 2017; 216: 92-7

5. Chan JK, Kawar NM, Shin JY, et al. Endometrial stromal sarcoma: a population-based analysis. Br J Cancer. 2008; 99(8): 1210-5.

6. Sleijfer S, Ouali M, van Glabbeke $\mathrm{M}$, et al. Prognostic and predictive factors for outcome to first-line ifosfamide-containing chemotherapy for adult patients with advanced soft tissue sarcomas: an exploratory, retrospective analysis on large series from the European organization for research and treatment of cancer-soft tissue and bone sarcoma group (EORTC-STBSG). Eur J Cancer. 2010; 46(1): 72-83.

7. Thanopoulou E, Aleksic A, Thway K, et al. Hormonal treatments in metastatic endometrial stromal sarcomas: the 10-year experience of the sarcoma unit of Royal Marsden Hospital. Clin Sarcoma Res. 2015; 5: 8.

8. Hoang L, Chiang S, Lee CH. Endometrial stromal sarcomas and related neoplasms: new developments and diagnostic considerations. Pathology. 2018; 50(2): 162-77.

9. Amant F, Floquet A, Friedlander M, et al. Gynecologic Cancer Inter Group (GCIG) consensus review for endometrial stromal sarcoma. Int J Gynecol Cancer. 2014; 24(9): S67-72.

10. Gadducci A, Cosio S, Romanini A, et al. The management of patients with uterine sarcoma: a debated clinical challenge. Crit Rev Oncol Hematol. 2008; 65(2): 129-42.

11. Reich $\mathrm{O}$, Regauer $\mathrm{S}$. Hormonal therapy of endometrial stromal sarcoma. Curr Opin Oncol. 2007; 19(4): 347-52

12. Machida H, Nathenson MJ, Takiuchi T, et al. Significance of lymph node metastasis on survival of women with uterine adenosarcoma. Gynecol Oncol. 2017; 144(3): 524-30.
13. Carroll A, Ramirez PT, Westin SN, et al. Uterine adenosarcoma: an analysis on management, outcomes, and risk factors for recurrence. Gynecol Oncol. 2014; 135(3): 455-61.

14. Grady D. Uterine surgical technique is linked to abnormal growths and cancer spread $[N]$. The New York Times. 2014-8-5.

15. Gao Z, Li L, Meng Y. A retrospective analysis of the Impact of myomectomy on survival in uterine sarcoma. PLoS One. 2016; 11(4): e0153996.

16. Li AJ, Giuntolind RL, Drake R, et al. Ovarian preservation in stage I low grade endometrial stromal sarcomas. Obstet Gynecol. 2005; 106(6): 1304-8.

17. Chu MC, Mor G, Lim C, et al. Low-grade endometrial stromal sarcoma: hormonal aspects. Gynecol Oncol. 2003; 90(1): 170-6.

18. Seagle BL, Shilpi A, Buchanan S, et al. Low-grade and high-grade endometrial stromal sarcoma: A National Cancer Database study. Gynecol Oncol. 2017; 146(2): 254-62.

19. Si M, Jia L, Song K, et al. Role of lymphadenectomy for uterine sarcoma: a meta-analysis. Int J Gynecol Cancer. 2017; 27(1): 109-16.

20. Benson $\mathrm{C}$, Miah $\mathrm{AB}$. Uterine sarcoma - current perspectives. Int J Womens Health. 2017; 31(9): 597-606.

21. Stewart LE, Beck TL, Giannakopoulos NV, et al. Impact of oophorectomy and hormone suppression in low grade endometrial stromal sarcoma: a multicenter review. Gynecol Oncol. 2018; 149(2): 297-300.

22. Dahhan T, Fons G, Buist MR, et al. The efficacy of hormonal treatment for residual or recurrent low-grade endometrial stromal sarcoma: A retrospective study. Eur J Obstet Gynecol Reprod Biol. 2009; 144(1): 80-4.

23. Beck TL, Singhal PK, Ehrenberg HM, et al. Endometrial stromal sarcoma: analysis of recurrence following adjuvant treatment. Gynecol Oncol. 2012; 125(1): 141-4.

24. Chu MC, Mor G, Lim C, et al. Low-grade endometrial stromal sarcoma: hormonal aspects. Gynecol Oncol. 2003; 90(1): 170-6.

25. Malouf GG, Duclos J, Rey A, et al. Impact of adjuvant treatment modalities on the management of patients with stages I-II endometrial stromal sarcoma. Ann Oncol. 2010; 21(10): 2102-6.

26. Amant F, De Knijf A, Van Calster B, et al. Clinical study investigating the role of lymphadenectomy, surgical castration and adjuvant hormonal treatment in endometrial stromal sarcoma. Br J Cancer. 2007; 97(9): 1194-9.

27. Tanner EJ, Toussaint $\mathrm{T}$, Leitao Jr MM, et al. Management of uterine adenosarcomas with and without sarcomatous overgrowth. Gynecol Oncol. 2013; 129(1): 140-4. 